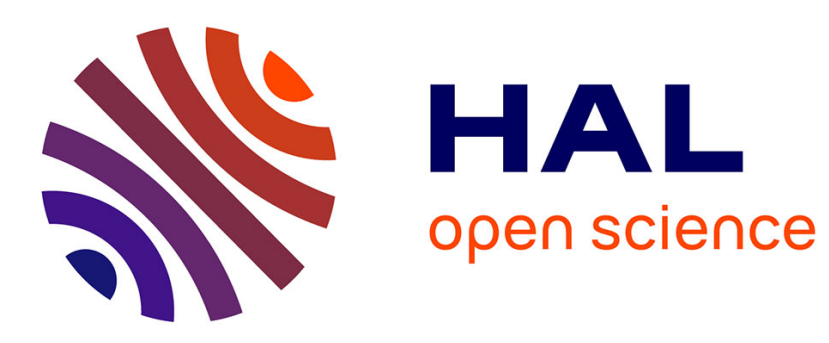

\title{
4-Valued Semantics Under the OWA: A Deductive Database Approach
}

Dominique Laurent

\section{To cite this version:}

Dominique Laurent. 4-Valued Semantics Under the OWA: A Deductive Database Approach. Information Search, Integration, and Personalization - 13th International Workshop, (ISIP) 2019, Heraklion, Greece, May 9-10, 2019, Revised Selected Papers, pp.101-116, 2020, 10.1007/978-3-030-44900-1_7 . hal-02986209

\section{HAL Id: hal-02986209 \\ https://hal.science/hal-02986209}

Submitted on 2 Nov 2020

HAL is a multi-disciplinary open access archive for the deposit and dissemination of scientific research documents, whether they are published or not. The documents may come from teaching and research institutions in France or abroad, or from public or private research centers.
L'archive ouverte pluridisciplinaire HAL, est destinée au dépôt et à la diffusion de documents scientifiques de niveau recherche, publiés ou non, émanant des établissements d'enseignement et de recherche français ou étrangers, des laboratoires publics ou privés. 


\title{
4-valued Semantics under the OWA: a Deductive Database Approach
}

\author{
Dominique Laurent \\ ETIS Laboratory - ENSEA / UCP / CNRS, Cergy-Pontoise, France \\ dominique. laurent@u-cergy.fr
}

\begin{abstract}
In this paper, we introduce a novel approach for dealing with databases containing inconsistent information. Considering four-valued logics in the context of OWA (Open World Assumption), a database $\Delta$ is a pair $(E, R)$ where $E$ is the extension and $R$ the set of rules. In our formalism, the set $E$ is a set of pairs of the form $\langle\varphi, v\rangle$ where $\varphi$ is a fact and $\mathrm{v}$ is either $\mathrm{t}$, or $\mathrm{b}$, or $\mathrm{f}$ (meaning respectively true, inconsistent or false), given that unknown facts are not stored. Moreover the rules extend Datalog ${ }^{\text {neg }}$ rules allowing their heads to be a negative atom.

We then define the notion of model of such a database, we show how to compute one particular model called semantics, and we investigate properties of this model. We also show how our approach applies to data integration and we review examples from the literature.
\end{abstract}

Key words: Open World Assumption . Datalog with negation . Inconsistent database . Database semantics.

\section{Introduction}

In this paper, we present and discuss our preliminary work on a novel approach meant to take into account the needs of many current applications, specifically in the domains of data integration and data warehousing:

1. As for usual Datalog databases ([6]), in our approach, a database $\Delta$ is a pair $(E, R)$ where $E$ (respectively $R$ ) is called the extension (respectively set of rules) of $\Delta$. Whereas in standard approaches $E$ is a set of ground facts meant to be true, in our approach $E$ is a set of pairs of the form $\langle\varphi, \mathrm{v}\rangle$ where $\varphi$ is a ground fact and $\mathrm{v}$ is one of the three truth values $\mathrm{t}$ (true), $\mathrm{b}$ (contradictory) or $f$ (false), meaning that a fact can be either true, or contradictory or false, given that facts not occurring in $E$ are considered unknown.

2. Recalling that a literal is an atom or the negation of an atom, the rules in $R$ are expressions of the form head $\leftarrow$ body where head is a literal and body is a conjunction (denoted as a list) of literals. We notice that such rules generalize standard Datalog ${ }^{\text {neg }}$ rules since their head may be a negated atom.

3 . The database semantics is defined based on the four valued semantics as defined in $[12,16]$. These semantics reflect the Open World Assumption (OWA), contrary to most database models that assume the Closed World Assumption (CWA) ([14]). As in [4], we argue that considering the OWA instead of 
the CWA is relevant in most applications related with data integration on the Web, for the following intuitive reason: when a fact does not appear in the answer to a query, this does not mean that this fact is false, but rather that it has not been searched properly. Therefore, in the absence of any other information such a fact will be considered unknown, instead of false.

As compared with related work on contradictions in databases, we propose a radically different approach. Indeed, the purpose of previous work dealing with contradictions in databases, is either to define and investigate 'repairs' so as to make the database consistent $([1,9])$, and/or to identify a set of queries whose answer is independent from any contradiction ([11]). Instead, we propose an approach in which contradictions can be stored or deduced through rules, and our purpose is not to eliminate or avoid contradictions. Instead, our semantics allows for handling contradictory information as such, thus reflecting real world applications in which true, false, inconsistent and unknown information have to be dealt with, as is the case when data integration is involved. In this context, the main contributions of this work are the following:

1. We define semantics for databases containing contradictions in the context of OWA. To do so, we review the formalism introduced in [3] and further discussed in the literature $[2,7,12,16]$, in particular regarding implication.

2. We study minimality properties of our semantics with respect to set inclusion and to the two orderings induced by four-valued logics (truth and knowledge orderings). We also relate our semantics to standard Datalog semantics ([6]).

3. We discuss the relevance of our approach in the context of data integration, a context of interest in more and more current applications. We illustrate this point by reviewing two well-known examples from the literature.

The paper is organized as follows: In Section 2 we recall some background on fourvalued logics and we introduce basic definitions. Section 3 deals with database semantics and minimality properties. In Section 4 , we review examples from the literature in the context of our approach. Section 5 concludes the paper.

\section{Background: Four-Valued Logics \& Database Context}

\subsection{Basics on Four-Valued Logics}

Four-valued logics was introduced by Belnap in [3], who argued that this formalism could be of interest when integrating data from various data sources. To this end, denoting by $t, b, n$ and $f$ the four truth values, the usual connectives $\neg, \vee$ and $\wedge$ have been defined as shown in Figure 1. An important feature of this four-valued logics is that it allows to compare truth values according to two partial orderings, known as truth ordering and knowledge ordering, respectively denoted by $\preceq_{t}$ and $\preceq_{k}$ and defined by:

$$
\mathrm{n} \preceq_{k} \mathrm{t} \preceq_{k} \mathrm{~b}, \mathrm{n} \preceq_{k} \mathrm{f} \preceq_{k} \mathrm{~b} \quad \text { and } \quad \mathrm{f} \preceq_{t} \mathrm{n} \preceq_{t} \mathrm{t}, \mathrm{f} \preceq_{t} \mathrm{~b} \preceq_{t} \mathrm{t} .
$$

As in standard two-valued logics, conjunction (respectively disjunction) corresponds to minimum (respectively maximum) truth value, when considering the 
truth ordering. It has also been shown in $[3,7]$ that the set $\{t, b, n, f\}$ equipped with these two orderings has a distributive bi-lattice structure. In this bi-lattice, the minimum and maximum with respect to $\preceq_{t}$ are $\wedge$ and $\vee$, and the minimum and maximum with respect to $\preceq_{k}$ are respectively denoted by $\otimes$ and $\oplus$.

This four-valued logics has motivated many comments and research, because some basic properties holding in standard logics do not hold in this formalism. For example, Figure 1 shows that formulas of the form $\Phi \vee \neg \Phi$ are not always true, independently from the truth value of $\Phi$. More importantly, it has been argued in $[2,12,16]$ that defining the implication $\Phi_{1} \Rightarrow \Phi_{2}$ as usual in two-valued logics, that is by $\neg \Phi_{1} \vee \Phi_{2}$, is problematic.

To see this, we consider as in $[2,3,12,16]$, that $\mathrm{t}$ and $\mathrm{b}$ are the two designated truth values, and a formula $\Phi$ is said to be valid if its truth value is designated. For instance, assuming that the truth value of $\Phi$ is $\mathrm{n}$, then $\Phi \Rightarrow \Phi$ is not valid since its truth value is $\mathrm{n}$. Notice in this respect that Figure 1 shows that $\Phi \rightarrow \Phi$, $\Phi \hookrightarrow \Phi, \Phi \stackrel{*}{\rightarrow} \Phi$ and $\Phi \stackrel{*}{\hookrightarrow} \Phi$ are valid for any truth assignment regarding $\Phi$.

As argued in $[2,12,16]$, considering $\Rightarrow$ as an implication does not satisfy the deduction theorem, because the formula $\Phi$ defined by $\left(\Phi_{1} \wedge\left(\Phi_{1} \Rightarrow \Phi_{2}\right)\right) \Rightarrow \Phi_{2}$ is not valid for any assignment $v$ such that $v\left(\Phi_{1}\right)=\mathrm{b}$ and $v\left(\Phi_{2}\right)=\mathrm{f}$ (because in such case, $v\left(\Phi_{1} \Rightarrow \Phi_{2}\right)=\mathrm{b}$ and thus, $\left.v(\Phi)=\mathrm{f}\right)$.

It can however be considered that Modus Ponens holds, in the sense that the statement [if $v\left(\Phi_{1}\right)$ and $v\left(\Phi_{1} \Rightarrow \Phi_{2}\right)$ are equal to $\mathrm{t}$ then $v\left(\Phi_{2}\right)=\mathrm{t}$ ] holds. However, this same statement enhanced by replacing 'true' with 'designated' does not hold because for $v\left(\Phi_{1}\right)=\mathrm{b}$ and $. v\left(\Phi_{2}\right)=\mathrm{f}$ we have $v\left(\Phi_{1} \Rightarrow \Phi_{2}\right)=\mathrm{b}$. This matter of fact is a severe limitation in our context, which explains why implication defined by ' $\Rightarrow$ ' is discarded as a possible semantics of our rules.

Other implications have been introduced, among which two in $[2,12]$ denoted hereafter by $\rightarrow$ and $\stackrel{*}{\rightarrow}$, and two in [16] denoted hereafter by $\hookrightarrow$ and $\stackrel{*}{\hookrightarrow}$. The truth tables of these implications are shown in Figure 1.

We recall from [2] (Corollary 9) that $\rightarrow$ is defined 'from scratch' in the sense that it cannot be expressed using the other standard connectives $\neg, \vee$ and $\wedge$. Moreover, since $\Phi_{1} \rightarrow \Phi_{2}$ and $\neg \Phi_{2} \rightarrow \neg \Phi_{1}$ are not equivalent, the implication $\Phi_{1} \stackrel{*}{\rightarrow} \Phi_{2}$ is introduced in $[2,12]$ as $\left(\Phi_{1} \rightarrow \Phi_{2}\right) \wedge\left(\neg \Phi_{2} \rightarrow \neg \Phi_{1}\right)$. The implication $\Phi_{1} \hookrightarrow \Phi_{2}$ is defined in [16] by $\sim \Phi_{1} \vee \Phi_{2}$, where $\sim$ is a complement operator whose truth table is shown in Figure 1. Again, $\Phi_{1} \hookrightarrow \Phi_{2}$ and $\neg \Phi_{2} \hookrightarrow \neg \Phi_{1}$ are not equivalent, and so, $\Phi_{1} \stackrel{*}{\hookrightarrow} \Phi_{2}$ is defined as $\left(\Phi_{1} \hookrightarrow \Phi_{2}\right) \wedge\left(\neg \Phi_{2} \hookrightarrow \neg \Phi_{1}\right)$.

Since the formula $\Phi$ defined by $\left(\Phi_{1} \wedge\left(\Phi_{1} \rightsquigarrow \Phi_{2}\right)\right) \rightsquigarrow \Phi_{2}$ is valid for any truth value assignment when replacing $\rightsquigarrow$ with one of the implications $\rightarrow, \hookrightarrow, \stackrel{*}{\rightarrow}$ or $\stackrel{*}{\hookrightarrow}$, Modus Ponens does apply in its standard and enhanced forms for any of these implications. It is also interesting to see that when merging the truth values $t$ and $b$ (respectively $f$ and $n$ ) into a single truth value, say True (respectively False), the corresponding truth tables of $\rightarrow$ and $\hookrightarrow$ are that of the standard implication, while this is not the case for $\Rightarrow, \stackrel{*}{\rightarrow}$ and $\stackrel{*}{\rightarrow}$. This explains why we discard these three implications. However, the choice between $\rightarrow$ and $\hookrightarrow$ is not easy for the following reasons: 


\begin{tabular}{|c|c|c|c|c|c|c|c|c|c|c|c|c|c|c|c|c|c|c|c|c|c|c|c|}
\hline$\varphi$ & $\neg \varphi$ & & & $\varphi$ & $\sim \varphi$ & & V & $t$ & $\mathrm{~b}$ & $\mathrm{n}$ & $f$ & & $\wedge$ & $t$ & $\mathrm{~b}$ & $\mathrm{n}$ & $\mathrm{f}$ & & $\Rightarrow$ & $t$ & $\mathrm{~b}$ & $\mathrm{n}$ & $f$ \\
\hline$t$ & $f$ & & & $t$ & $f$ & & $t$ & $t$ & $t$ & $t$ & $t$ & & $t$ & $t$ & $\mathrm{~b}$ & $\mathrm{n}$ & $f$ & & $t$ & $t$ & $\mathrm{~b}$ & $n$ & $f$ \\
\hline $\mathrm{b}$ & $\mathrm{b}$ & & & $\mathrm{b}$ & $\mathrm{n}$ & & $\mathrm{b}$ & $t$ & $\mathrm{~b}$ & $t$ & $b$ & & $\mathrm{~b}$ & $\mathrm{~b}$ & $\mathrm{~b}$ & $f$ & $f$ & & b & $t$ & $\mathrm{~b}$ & $t$ & $\mathrm{~b}$ \\
\hline $\mathrm{n}$ & $\mathrm{n}$ & & & $\mathrm{n}$ & b & & $\mathrm{n}$ & $t$ & $t$ & $\mathrm{n}$ & $\mathrm{n}$ & & $\mathrm{n}$ & $\mathrm{n}$ & $f$ & $\mathrm{n}$ & $f$ & & $\mathrm{n}$ & $t$ & $t$ & $\mathrm{n}$ & n \\
\hline$f$ & $t$ & & & $f$ & $t$ & & $f$ & $t$ & $b$ & $\mathrm{n}$ & $f$ & & $\mathrm{f}$ & $\mathrm{f}$ & $\mathrm{f}$ & $\mathrm{f}$ & $\mathrm{f}$ & & $f$ & $t$ & $t$ & $t$ & t \\
\hline & $\rightarrow$ & $t$ & $\mathrm{~b}$ & $\mathrm{n}$ & $f$ & $\hookrightarrow$ & $t$ & $\mathrm{~b}$ & $\mathrm{n}$ & $f$ & & $\stackrel{*}{\rightarrow}$ & $t$ & b & $\mathrm{n}$ & $f$ & & $\stackrel{*}{\hookrightarrow}$ & $t$ & $\mathrm{~b}$ & $\mathrm{n}$ & $f$ & \\
\hline & $t$ & $t$ & $\mathrm{~b}$ & $\mathrm{n}$ & $f$ & $t$ & $t$ & $\mathrm{~b}$ & $\mathrm{n}$ & & & $t$ & $t$ & $f$ & $\mathrm{n}$ & $f$ & & $t$ & $t$ & $f$ & $f$ & $\mathrm{f}$ & \\
\hline & $\mathrm{b}$ & $t$ & $\mathrm{~b}$ & $n$ & $f$ & $\mathrm{~b}$ & $t$ & $t$ & $\mathrm{n}$ & $\mathrm{n}$ & & $b$ & $t$ & $b$ & $\mathrm{n}$ & $f$ & & $\mathrm{~b}$ & $t$ & $t$ & $f$ & $f$ & \\
\hline & $\mathrm{n}$ & $t$ & $t$ & $t$ & $t$ & $\mathrm{n}$ & $t$ & $\mathrm{~b}$ & $t$ & $b$ & & $\mathrm{n}$ & $t$ & $\mathrm{n}$ & $t$ & $\mathrm{n}$ & & $\mathrm{n}$ & $t$ & $f$ & $t$ & $f$ & \\
\hline & $f$ & $t$ & $t$ & $t$ & $t$ & $f$ & $t$ & $t$ & $t$ & $t$ & & $\mathrm{f}$ & $t$ & $t$ & $t$ & $t$ & & $f$ & $t$ & $\mathrm{t}$ & $\mathrm{t}$ & $t$ & \\
\hline
\end{tabular}

Fig. 1. Truth tables of basic connectors and implications

- In $[2,12]$, it is argued that, similarly to two-valued implication, $\rightarrow$ satisfies the property that $v\left(\Phi_{1} \rightarrow \Phi_{2}\right)=v\left(\Phi_{2}\right)$ whenever $v\left(\Phi_{1}\right)$ is designated. However, $\rightarrow$ does not satisfy the properties of $\hookrightarrow$ given below.

- Although $\hookrightarrow$ does not satisfy the above property, it is argued in [16] that, similarly to two-valued implication, $\hookrightarrow$ satisfies the property that $v\left(\Phi_{1}\right) \preceq_{t}$ $v\left(\Phi_{2}\right)$ if and only if $v\left(\Phi_{1} \hookrightarrow \Phi_{2}\right)=\mathrm{t}$.

We draw attention on that none of these two implications does satisfy all intuitively appealing properties that standard two-valued implication satisfies, among which contraposition is an important example. Since in our approach, implications are seen as rules, Modus Ponens is the basic 'logical tool' to be used, whereas contraposition is not used as such.

Looking at the truth tables of the two implications $\rightarrow$ and $\hookrightarrow$ shown in Figure 1, when the left hand side is valid in $S$, it is necessary that the right hand side be also valid in order to make the implication valid. More precisely, if $\Phi_{1}$ is valid, the implications $\Phi_{1} \rightarrow \Phi_{2}$ and $\Phi_{1} \hookrightarrow \Phi_{2}$ are valid in $S$ for any truth assignment $v$ such that:

$-v\left(\Phi_{1}\right)=\mathrm{t}$ and $v\left(\Phi_{2}\right)=\mathrm{t}$ or $v\left(\Phi_{2}\right)=\mathrm{b}$,

$-v\left(\Phi_{1}\right)=\mathrm{b}$ and $v\left(\Phi_{2}\right)=\mathrm{t}$ or $v\left(\Phi_{2}\right)=\mathrm{b}$.

As a consequence, if it happens that $\Phi_{1}$ is valid while $\Phi_{2}$ is not, the implication can be made valid by changing the truth value of $\Phi_{2}$ in two ways: making it either true or inconsistent. As will be seen later, we choose to set $v_{S}\left(\Phi_{2}\right)$ as equal to $v_{S}\left(\Phi_{1}\right)$. This choice is motivated by the fact that it is the only one satisfying $v\left(\Phi_{1}\right) \preceq_{k} v\left(\Phi_{2}\right)$ and $v\left(\Phi_{1}\right) \preceq_{t} v\left(\Phi_{2}\right)$.

\subsection{Four-Valued Logics in the Database Context}

As usual when dealing with deductive databases, the considered alphabet is made of constants, variables and predicate symbols, and ground atoms are called facts. We thus assume a fixed set of facts, called universe and denoted by $\mathcal{U}$. The set $\mathcal{U}$ is the set of all possible facts that can be built up using the constants and 
predicates that occur in the database being modeled. It is therefore important to notice that, as databases are assumed to be finite, so is $\mathcal{U}$.

While in the two-valued setting under the CWA, the database extension and the database semantics are sets of facts, meant to be true and the facts not occurring in the database semantics are set to be false, in our context of fourvalued logics under the OWA, the database extension and the database semantics may contain facts that are either true, contradictory or false, assuming that non stored facts are unknown.

To account for this situation, we consider sets of pairs $\langle\varphi, v\rangle$ where $\varphi$ is a fact in $\mathcal{U}$ and where $\mathrm{v}$ is one of the truth values $t, \mathrm{~b}, \mathrm{n}$ or $\mathrm{f}$, and we assume that facts whose truth value is n are not stored. Such a set $S$ is said to be consistent if for all distinct pairs $\left\langle\varphi_{1}, \mathrm{v}_{1}\right\rangle$ and $\left\langle\varphi_{2}, \mathrm{v}_{2}\right\rangle$ in $S, \varphi_{1} \neq \varphi_{2}$. Consequently a consistent set $S$ is seen as a valuation $v_{S}$ defined for every $\varphi$ in $\mathcal{U}$ by:

$$
v_{S}(\varphi)=\mathrm{v} \text {, if } S \text { contains a pair }\langle\varphi, \mathrm{v}\rangle ; v_{S}(\varphi)=\mathrm{n} \text {, otherwise. }
$$

Consistent sets of pairs are called v-sets, standing for valuated sets. It should be noticed that, in [16], a formal way of expressing the pairs in a v-set is proposed by defining unary operators. We however do not use this formalism because its proper definition requires further notation which could be thought unnecessarily sophisticated in our context.

Given a v-set $S$ and a formula $\Phi$, based on the truth tables given in Figure 1, $\Phi$ is said to be valid in $S$ if $v_{S}(\Phi)$ is designated. For example, $a \rightarrow b$ is valid in $S_{1}=\{\langle a, \mathrm{t}\rangle,\langle b, \mathrm{~b}\rangle\}$ because $v_{S_{1}}(a \rightarrow b)=\mathrm{b}$, but $a \rightarrow b$ is not valid in $S_{2}=\{\langle a, \mathrm{t}\rangle\}$ because $v_{S_{2}}(a \rightarrow b)=\mathrm{n}$.

The two orderings $\preceq_{k}$ and $\preceq_{t}$ are extended to v-sets over the same universe $\mathcal{U}$ as follows.

Definition 1. For all v-sets $S_{1}$ and $S_{2}$ over $\mathcal{U}, S_{1} \preceq_{k} S_{2}$, respectively $S_{1} \preceq_{t} S_{2}$, holds if for every $\varphi$ in $\mathcal{U}, v_{S_{1}}(\varphi) \preceq_{k} v_{S_{2}}(\varphi)$, respectively $v_{S_{1}}(\varphi) \preceq_{t} v_{S_{2}}(\varphi)$, holds.

For example with $\mathcal{U}=\{a, b, c\}, S_{1}=\{\langle a, \mathrm{t}\rangle\}$ and $S_{2}=\{\langle a, \mathrm{~b}\rangle,\langle b, \mathrm{f}\rangle\}$, we have $v_{S_{1}}(b)=v_{S_{1}}(c)=v_{S_{2}}(c)=\mathrm{n}$ and so:

$-v_{S_{1}}(a) \preceq_{k} v_{S_{2}}(a), v_{S_{1}}(b) \preceq_{k} v_{S_{2}}(b)$ and $v_{S_{1}}(c) \preceq_{k} v_{S_{2}}(c)$, implying that $S_{1} \preceq_{k} S_{2}$ holds.

- $v_{S_{2}}(a) \preceq_{t} v_{S_{1}}(a), v_{S_{2}}(b) \preceq_{t} v_{S_{1}}(b)$ and $v_{S_{2}}(c) \preceq_{t} v_{S_{1}}(c)$, implying that $S_{2} \preceq_{t}$ $S_{1}$ holds.

- $\emptyset \preceq_{k} S_{2}$, because for every $\varphi, v_{\emptyset}(\varphi)=\mathrm{n}$, the least value with respect to $\preceq_{k}$.

- $\emptyset$ and $S_{2}$ are not comparable with respect to $\preceq_{t}$, because $v_{\emptyset}(a)=\mathrm{n}$ and $v_{S_{2}}(a)=\mathrm{b}$ are not comparable with respect to $\preceq_{t}$.

The extension of $\preceq_{k}$ generalizes set inclusion in the sense that if $S_{1} \subseteq S_{2}$, then we have $S_{1} \preceq_{k} S_{2}$. Notice that, as the last item above shows, the truth ordering $\preceq_{t}$ does not satisfy this property, because $\emptyset \subseteq S_{2}$ holds while $\emptyset \preceq_{t} S_{2}$ does not.

\section{Database and Database Semantics}

As in standard approaches to Datalog databases $([5,6])$, a database consists of an extension and a set of rules, formally defined as follows. 
Definition 2. A database $\Delta$ is a pair $\Delta=(E, R)$ where $E$ and $R$ are respectively called the extension and the rule set of $\Delta$. If $\Delta=(E, R)$, then:

- E is a v-set.

$-R$ is a set of rules of the form $\rho: h \leftarrow b_{1}, \ldots, b_{n}$ where

1. for $i=1, \ldots, n, b_{i}$ is a literal (positive or negative) and the set of all $b_{i}$ 's $(i=1, \ldots, n)$ is called the body of $\rho$, denoted by body $(\rho)$,

2. $h$ is a positive or negative literal, called the head of $\rho$, denoted by head $(\rho)$,

3. all variables occurring in $h$ also occur in body $(\rho)$, i.e., rules are safe.

It is important to notice that the rules in our approach generalize Datalog ${ }^{n e g}$ rules ([5]) because negative atoms are allowed not only in the body but also in the head of the rules. This implies that rules may generate contradictory facts.

As usual, rules are seen as implications, either $\rightarrow$ or $\hookrightarrow$ that must be valid in the database semantics. Notice in this respect that Figure 1 shows that for all formulas $\phi_{1}$ and $\phi_{2}, \phi_{1} \rightarrow \phi_{2}$ is valid if and only if so is $\phi_{1} \hookrightarrow \phi_{2}$. This explains why our approach can be said 'compatible' with either implication.

Similarly to the standard Datalog approach, a model of a database $\Delta=$ $(E, R)$ could be defined as a v-set $M$ containing $E$ and in which all rules in $R$ are valid. However, such a definition would raise important problems:

1. A database might have no model. To see this, consider $\Delta=(E, R)$ where $R=$ $\{b \leftarrow a\}$ and where $E=\{\langle a, \mathrm{t}\rangle,\langle b, \mathbf{f}\rangle\}$. Whatever the chosen implication (either $\rightarrow$ or $\hookrightarrow$ ), in any model $M, v_{M}(a \rightarrow b)=v_{M}(a \hookrightarrow b)=\mathrm{f}$ because $M$ must contain the two pairs of $E$. Notice that this cannot happen in standard Datalog since the storage of false facts is not allowed.

2. A database might have more than one minimal model, with respect to set inclusion. This case is illustrated above where $S_{1}^{\prime}=\{\langle a, \mathrm{t}\rangle,\langle b, \mathrm{t}\rangle\}$ are $S_{2}^{\prime}=$ $\{\langle a, \mathrm{t}\rangle,\langle b, \mathrm{~b}\rangle\}$ two minimal v-sets containing $\{\langle a, \mathrm{t}\rangle\}$ in which $b \leftarrow a$ is valid. This situation does not happen in standard Datalog because the minimal model is known to be unique.

Whereas the second issue raised above will be further investigated later, the first issue is solved in our approach by giving the priority to the database extension over the rules. To do so, we prevent from applying a rule in $R$ when it leads to some conflict with a pair in $E$. In order to implement this policy, given a database $\Delta=(E, R)$ over universe $\mathcal{U}$, we denote by $\operatorname{inst}(E, R)$ the set of all instantiations $\rho$ of rules in $R$ such that $h e a d(\rho)$ does not occur in $E$. Moreover, given a rule $\rho: h \leftarrow b$ we denote by $\rho^{\rightarrow}$, respectively $\rho^{\hookrightarrow}$, the formula $b \rightarrow h$, respectively $b \hookrightarrow h$. The definition of a model of $\Delta$ then follows.

Definition 3. Let $\Delta=(E, R)$ be a database over universe $\mathcal{U}$. $A$ v-set $M$ is a model of $\Delta$ if the following holds:

1. $E \subseteq M$, i.e., $M$ must contain the database extension, and

2. every $\rho$ of $\operatorname{inst}(E, R)$ is valid in $M$, i.e., $v_{M}\left(\rho^{\rightarrow}\right)$ and $v_{M}\left(\rho^{\hookrightarrow}\right)$ are designated.

Referring to the previous two items, Definition 3 applies as follows: 
- For $\Delta=(E, R)$ with $E=\{\langle a, \mathrm{t}\rangle,\langle b, \mathrm{f}\rangle\}$ and $R=\{b \leftarrow a\}, E$ is a model of $\Delta$ since we have $\operatorname{inst}(E, R)=\emptyset$. Notice that here, $E$ is the only minimal model with respect to set inclusion.

- For $\Delta=(E, R)$ with $E=\{\langle a, \mathrm{t}\rangle\}$ and $R=\{b \leftarrow a\}, S_{1}^{\prime}=\{\langle a, \mathrm{t}\rangle,\langle b, \mathrm{t}\rangle\}$ are $S_{2}^{\prime}=\{\langle a, \mathrm{t}\rangle,\langle b, \mathrm{~b}\rangle\}$ two minimal models of $\Delta$, because inst $(E, R)=R$ and the two v-sets satisfy Definition 3 .

Given a database $\Delta$, a modified version of the membership immediate consequence operator $([5,7])$ is defined below. It will then be seen that this allows for computing a particular model of $\Delta$, which we call the semantics of $\Delta$.

Definition 4. Let $\Delta=(E, R)$ be a database. The semantic consequence operator associated with $\Delta$, denoted by $\Sigma_{\Delta}$, is defined for every v-set $S$ by the following steps:

(1) Define first $\Gamma_{\Delta}^{E}(S)$ as follows:

$$
\begin{aligned}
\Gamma_{\Delta}^{E}(S)=E & \cup\left\{\langle h, \mathrm{t}\rangle \mid(\exists \rho \in \operatorname{inst}(E, R))\left(h=\operatorname{head}(\rho) \wedge v_{S}(\operatorname{body}(\rho))=\mathrm{t}\right)\right\} \\
& \cup\left\{\langle h, \mathrm{~b}\rangle \mid(\exists \rho \in \operatorname{inst}(E, R))\left(h=\operatorname{head}(\rho) \wedge v_{S}(\operatorname{body}(\rho))=\mathrm{b}\right)\right\} \\
& \cup\left\{\langle h, \mathbf{f}\rangle \mid(\exists \rho \in \operatorname{inst}(E, R))\left(\neg h=\operatorname{head}(\rho) \wedge v_{S}(\operatorname{body}(\rho))=\mathrm{t}\right)\right\} \\
& \cup\left\{\langle h, \mathrm{~b}\rangle \mid(\exists \rho \in \operatorname{inst}(E, R))\left(\neg h=\operatorname{head}(\rho) \wedge v_{S}(\operatorname{body}(\rho))=\mathrm{b}\right)\right\}
\end{aligned}
$$

(2) Then, $\Sigma_{\Delta}(S)$ is defined by:

$$
\Sigma_{\Delta}(S)=\left\{\left\langle\varphi, \mathrm{v}_{\oplus}(\varphi)\right\rangle \mid \varphi \text { occurs in } \Gamma_{\Delta}^{E}(S)\right\}
$$

where $\mathrm{v}_{\oplus}(\varphi)=\max _{k}\left\{\mathrm{v} \mid\langle\varphi, \mathrm{v}\rangle \in \Gamma_{\Delta}^{E}(S)\right\}$.

The following lemma, whose proof can found in Appendix A, shows basic properties of the operator $\Sigma_{\Delta}$.

Lemma 1. For every $\Delta=(E, R)$ and all v-sets $S, S_{1}$ and $S_{2}$ :

1. $\Sigma_{\Delta}(S)$ is a v-set such that $E \preceq_{k} \Sigma_{\Delta}(S)$.

2. If $S_{1} \preceq_{k} S_{2}$, then $\Sigma_{\Delta}\left(S_{1}\right) \preceq_{k} \Sigma_{\Delta}\left(S_{2}\right)$.

As a consequence of Lemma 1 , with respect to $\preceq_{k}$, the sequence defined by

$-\Sigma^{0}=E$

- for every $n \geq 1, \Sigma^{n}=\Sigma_{\Delta}\left(\Sigma^{n-1}\right)$

is monotonic, that is for every $i \geq 0, \Sigma^{i} \preceq_{k} \Sigma^{i+1}$, and since $\mathcal{U}$ is finite, this sequence has a unique limit. We denote this limit as $\Sigma_{\Delta}^{*}$ and we call it the semantics of $\Delta$. Moreover, the valuation $v_{\Sigma_{\Delta}^{*}}$ is more simply denoted by $v_{\Delta}$ and the valid facts in $\Sigma_{\Delta}^{*}$ are said to be valid in $\Delta$.

As a consequence of the above properties, it should be clear that $\Sigma_{\Delta}^{*}$ is a vset such that $E \subseteq \Sigma_{\Delta}^{*}$ and $E \preceq_{k} \Sigma_{\Delta}^{*}$. This intuitively means that the semantics extends the content and the knowledge provided by the database extension $E$. The following example illustrates cases of computation of $\Sigma_{\Delta}^{*}$.

Example 1. Consider the database $\Delta=(E, R)$ over $\mathcal{U}=\{a, b, c, d, e\}$ where $R$ is the set of the following three rules

$$
\rho_{1}: c \leftarrow a, \neg b \quad ; \quad \rho_{2}: e \leftarrow d \quad ; \quad \rho_{3}: c \leftarrow e
$$

and where $E=\{\langle a, \mathrm{t}\rangle,\langle b, \mathbf{f}\rangle,\langle e, \mathrm{~b}\rangle\}$. In this case, inst $(E, R)=\left\{\rho_{1}, \rho_{3}\right\}$ and the computation of $\Sigma_{\Delta^{\prime}}^{*}$ is as follows: 
1. $\Sigma^{1}=E$.

2. $\Sigma^{1}=\Sigma_{\Delta^{\prime}}\left(\Sigma^{0}\right)$. We have $\Gamma_{\Delta^{\prime}}^{\in}\left(\Sigma^{0}\right)=E \cup\{\langle c, \mathrm{t}\rangle,\langle c, \mathrm{~b}\rangle\}$, which is clearly not an acceptable result since it is not a proper v-set. When computing $\Sigma^{1}$, the first pair is removed, and we obtain that $\Sigma^{1}=E \cup\{\langle c, \mathrm{~b}\rangle\}$.

3. Since $\Sigma^{2}=\Sigma^{1}$ (no rule applies on $\Sigma^{1}$ to produce new pairs), the computation stops returning that $\Sigma_{\Delta}^{*}=\Sigma^{1}=\{\langle a, \mathrm{t}\rangle,\langle b, \mathrm{f}\rangle,\langle c, \mathrm{~b}\rangle,\langle e, \mathrm{~b}\rangle\}$.

We draw attention on that $E$ and $\Sigma_{\Delta}$ are not comparable with respect to $\preceq_{t}$ because $v_{E}(c)=\mathrm{n}$ and $v_{\Delta}(c)=\mathrm{b}$. Hence, $E \preceq_{t} \Sigma_{\Delta}$ does not hold in general, contrary to $E \preceq_{k} \Sigma_{\Delta}$. Moreover, $\Sigma_{\Delta}^{*}$ is a model of $\Delta$ because $E \subseteq \Sigma_{\Delta}^{*}$ and:

$-\rho_{1}$ and $\rho_{1}^{\hookrightarrow}$ are valid in $\Sigma_{\Delta}^{*}$ because $v_{\Delta}\left(\rho_{1}^{\rightarrow}\right)=v_{\Delta}\left(\rho_{1}^{\hookrightarrow}\right)=\mathrm{b}$, due to the fact that $v_{\Delta}(a \wedge \neg b)=\mathrm{t}$ and $v_{\Delta}(c)=\mathrm{b}$.

$-\rho_{2}$ and $\rho_{2}^{\hookrightarrow}$ are also valid in $\Sigma_{\Delta}^{*}$. Indeed, as $v_{\Delta}(d)=\mathrm{n}$ and $v_{\Delta}(e)=\mathrm{b}$, we have $v_{\Delta}\left(\rho_{2}\right)=\mathrm{t}$ and $v_{\Delta}\left(\rho_{2}^{\hookrightarrow}\right)=\mathrm{b}$.

$-\rho_{3}$ and $\rho_{3}^{\hookrightarrow}$ are valid in $\Sigma_{\Delta}^{*}$ as well. Indeed, as $v_{\Delta}(e)=v_{\Delta}(c)=\mathrm{b}$, we have $v_{\Delta}\left(\rho_{3}\right)=\mathrm{b}$ and $v_{\Delta}\left(\rho_{3}^{\hookrightarrow}\right)=\mathrm{t}$.

The following proposition, whose proof can be found in Appendix B, shows that, as seen in Example 1, $\Sigma_{\Delta}^{*}$ is a minimal model of $\Delta$ with respect to set inclusion.

Proposition 1. Given a database $\Delta=(E, R), \Sigma_{\Delta}^{*}$ is a minimal (with respect to set inclusion) model of $\Delta$.

However, the following example shows that $\Sigma_{\Delta}^{*}$ is not the only minimal model with respect to set inclusion and that $\Sigma_{\Delta}^{*}$ is neither minimal nor maximal with respect to any of the two orderings $\preceq_{k}$ and $\preceq_{t}$.

Example 2. Considering, as in Example 1, $\Delta=(E, R)$ where $E=\{\langle a, \mathrm{t}\rangle,\langle b, \mathrm{f}\rangle$, $\langle e, \mathrm{~b}\rangle\}$ and $R=\left\{\rho_{1}, \rho_{2}, \rho_{3}\right\}$, we recall that $\Sigma_{\Delta}^{*}=\{\langle a, \mathrm{t}\rangle,\langle b, \mathrm{f}\rangle,\langle c, \mathrm{~b}\rangle,\langle e, \mathrm{~b}\rangle\}$. Let $S$ and $S^{\prime}$ be the following v-sets :

$S=\{\langle a, \mathrm{t}\rangle,\langle b, \mathrm{f}\rangle,\langle c, \mathrm{t}\rangle,\langle e, \mathrm{~b}\rangle\} \quad$ and $S^{\prime}=\{\langle a, \mathrm{t}\rangle,\langle b, \mathrm{f}\rangle,\langle c, \mathrm{~b}\rangle,\langle d, \mathrm{f}\rangle,\langle e, \mathrm{~b}\rangle\}$. We first show that $S$ and $S^{\prime}$ are two models of $\Delta$. Indeed:

$-E \subseteq S$ and $E \subseteq S^{\prime}$.

- $\rho_{1} \overrightarrow{\text { and }} \rho_{1}^{\hookrightarrow}$ are valid in $S$ and $S^{\prime}$ because $v_{S}\left(\rho_{1}^{\overrightarrow{1}}\right)=v_{S}\left(\rho_{1}^{\hookrightarrow}\right)=v_{S^{\prime}}\left(\rho_{1}^{\overrightarrow{1}}\right)=$ $v_{S^{\prime}}\left(\rho_{1}^{\hookrightarrow}\right)=\mathrm{t}$, since $v_{S}(a \wedge \neg b)=v_{S^{\prime}}(a \wedge \neg b)=v_{S}(c)=v_{S^{\prime}}(c)=\mathrm{t}$.

$-\rho_{2} \vec{a}$ and $\rho_{2}^{\hookrightarrow}$ are not tested since $\rho_{2}$ is not in $\operatorname{inst}(E, R)$.

$-\rho_{3}$ and $\rho_{3}^{\hookrightarrow}$ are valid in $S$ and in $S^{\prime}$. Indeed, as $v_{S}(e)=\mathrm{b}$ and $v_{S}(c)=\mathrm{t}$, we have $v_{S}\left(\rho_{3}\right)=v_{S}\left(\rho_{3}^{\hookrightarrow}\right)=\mathrm{t}$, and since $v_{S^{\prime}}(e)=\mathrm{b}$ and $v_{S^{\prime}}(c)=\mathrm{b}$, we have $v_{S^{\prime}}\left(\rho_{3}\right)=\mathrm{b}$ and $v_{S^{\prime}}\left(\rho_{3}^{\hookrightarrow}\right)=\mathrm{t}$.

It can be seen that $\Sigma_{\Delta}$ is not the unique minimal model of $\Delta$ with respect to set inclusion, because $S$ is also such a minimal model, due to the fact that no proper subset of $S$ does satisfy Definition 3. Notice also that $S^{\prime}$ is not minimal with respect to set inclusion, because $\Sigma_{\Delta}^{*} \subset S^{\prime}$ holds.

Regarding minimality or maximality of $\Sigma_{\Delta}^{*}$ with respect to $\preceq_{t}$ or $\preceq_{k}$, we emphasize the following: 
- We have $S \prec_{k} \Sigma_{\Delta}^{*}$ and $\Sigma_{\Delta}^{*} \prec_{t} S$, because for every $\varphi$ in $\mathcal{U}$ different from $c$, $v_{S}(\varphi)=v_{\Delta}(\varphi), v_{S}(c) \prec_{k} v_{\Delta}(c)$, and $v_{\Delta}(c) \prec_{t} v_{S}(c)$. Therefore, $\Sigma_{\Delta}^{*}$ is not minimal with respect to $\prec_{k}$ and not maximal with respect to $\prec_{t}$.

- We have $S^{\prime} \prec_{t} \Sigma_{\Delta}^{*}$ and $\Sigma_{\Delta}^{*} \prec_{k} S^{\prime}$, because for every $\varphi$ in $\mathcal{U}$ different from $d$, $v_{S^{\prime}}(\varphi)=v_{\Delta}(\varphi)$ and $v_{S^{\prime}}(d)=\mathrm{f}, v_{\Delta}(d)=\mathrm{n}$, implying that $v_{S^{\prime}}(d) \prec_{t} v_{\Delta}(d)$ and that $v_{\Delta}(d) \prec_{k} v_{S^{\prime}}(d)$. Therefore, $\Sigma_{\Delta}^{*}$ is not minimal with respect to $\prec_{t}$ and not maximal with respect to $\prec_{k}$.

If $\Delta=(E, R)$ is a Datalog database, i.e., all facts are associated with $\mathrm{t}$ in $E$ and all literals in the rules in $R$ are positive, then it is easy to see that $\Sigma_{\Delta}^{*}=\left\{\langle\varphi, \mathrm{t}\rangle \mid \varphi \in M_{2}\right\}$, where $M_{2}$ is the unique minimal model of $\Delta$, as computed in Datalog. Thus, the true facts in $\Delta$ are the same as those, when considering $\Delta$ as a Datalog database. Notice however that for any $\varphi$ not in $M_{2}$, $\varphi$ is false in the Datalog approach, and $\varphi$ is unknown in our approach.

Considering now the more generic case where the rules are Datalog ${ }^{\text {neg }}$ rules and where false facts are allowed in $E$, the following proposition, whose proof is shown in Appendix $\mathrm{C}$, shows that in this case, all minimal models of $\Delta$ have the same valid facts and the same false facts. To this end, given a v-set $S$, we denote by $\mathrm{V}(S)$, respectively $\mathrm{F}(S)$, the set of all facts $\varphi$ that are valid in $S$ (i.e., such that $v_{S}(\varphi)=\mathrm{t}$ or $\left.v_{S}(\varphi)=\mathrm{b}\right)$, respectively false in $S\left(i . e\right.$., such that $\left.v_{S}(\varphi)=\mathrm{f}\right)$.

Proposition 2. Let $\Delta=(E, R)$ be such that for every rule $\rho$ in $R$, head $(\rho)$ is a positive literal. For all minimal models $M_{1}$ and $M_{2}$ of $\Delta$, the following holds: (i) $\mathrm{V}\left(M_{1}\right)=\mathrm{V}\left(M_{2}\right)$ and $(i i) \mathrm{F}\left(M_{1}\right)=\mathrm{F}\left(M_{2}\right)$.

\section{Application to Data Integration}

\subsection{The Generic Scenario}

Data integration is a generic context where our approach can be found useful. Indeed, assuming that $p$ data sources, i.e., $p$ databases, are to be integrated in one database, it may happen that two distinct data sources contain contradictory pieces of information. Formally, the integrated database extension is defined by a valuation $v$ defined as follows for every fact $\varphi$ :

$-v(\varphi)=\mathrm{t}$ if $\varphi$ is true in all sources providing information about $\varphi$.

$-v(\varphi)=\mathrm{b}$ if $\varphi$ is true in some sources and false in some other sources.

$-v(\varphi)=\mathrm{f}$ if $\varphi$ is false in all sources providing information about $\varphi$.

$-v(\varphi)=\mathrm{n}$ if no data sources provide information about $\varphi$.

In other words, assuming that for $i=1, \ldots, p$, the extension of the $i$ th data source is defined by a valuation $v_{i}$, the valuation $v$ defining the integrated database is defined for every fact $\varphi$ by: $v(\varphi)=\max _{k}\left\{v_{i}(\varphi) \mid i=1, \ldots, p\right\}$. Next, we review two examples from the literature in the framework of this scenario.

\subsection{The Case of Nixon Diamond}

This example deals with the following: on the one hand, quakers are known to be doves and republican are known to be hawks, and on the other hand, being 
a dove is not compatible with being a hawk. Knowing that Nixon (President of the USA, in the seventies) was a quaker and a republican, the question is: Was Nixon a hawk or a dove? The corresponding 'program' is as follows:

$$
\begin{gathered}
\text { dove }(x) \leftarrow \text { quaker }(x) ; \operatorname{hawk}(x) \leftarrow \operatorname{republican}(x) \\
\neg \text { dove }(x) \leftarrow \operatorname{hawk}(x) ; \neg \operatorname{hawk}(x) \leftarrow \operatorname{dove}(x) \\
\text { quaker }(\text { Nixon }) ; \operatorname{republican}(\text { Nixon })
\end{gathered}
$$

Notice that this can not be seen as a Datalog ${ }^{n e g}$ program, because of the third and fourth rules. In the literature this program has been the subject of many comments, and one of the most common approaches is known as stable semantics [8]. According to this approach, two minimal models are found: one asserting that Nixon is a quaker and thus dove, and the other one asserting that Nixon is a republican and thus a hawk.

In our approach, this can be thought of as stemming from two sources $S_{1}=\{\langle q u a k e r(N i x o n), \mathrm{t}\rangle\}$ and $S_{2}=\{\langle\operatorname{republican}($ Nixon $), \mathrm{t}\rangle\}$. According to the above generic scenario, the integrated database $\Delta=(E, R)$ is such that $E=S_{1} \cup S_{2}$ and $R$ contains the four rules displayed above. It is then easy to see that $\Sigma_{\Delta}$ contains $\langle\operatorname{dove}(N$ ixon), b $\rangle$ and $\langle$ hawk(Nixon), b $\rangle$, meaning that dove(Nixon) and hawk(Nixon) are contradictory.

A slightly different scenario of integration is to consider that the sources are aware of the rules and thus that they are defined by

$-S_{1}^{\prime}=\{\langle$ quaker(Nixon), $\mathrm{t}\rangle,\langle$ dove(Nixon), $\mathrm{t}\rangle,\langle$ hawk(Nixon), $\mathrm{f}\rangle\}$ and

$-S_{2}^{\prime}=\{\langle$ republican(Nixon), $\mathrm{t}\rangle,\langle$ hawk(Nixon), $\mathrm{t}\rangle,\langle$ dove(Nixon $\left.), \mathrm{f}\rangle\right\}$.

The integrated database $\Delta^{\prime}=\left(E^{\prime}, R\right)$ is such that $E^{\prime}=\{\langle$ quaker(Nixon), $\mathrm{t}\rangle$, $\langle$ republican(Nixon), t $\rangle,\langle$ hawk(Nixon), b $\rangle,\langle\operatorname{dove}(N i x o n), \mathrm{b}\rangle\}$, which leads as above to the fact that dove(Nixon) and hawk(Nixon) are contradictory.

As an extension to this example, some authors additionally consider that hawks and doves are politically motivated, and study the question: Is Nixon politically motivated? Formally two rules are added to the above program, namely $p m(x) \leftarrow \operatorname{dove}(x)$ and $p m(x) \leftarrow \operatorname{hawk}(x)$, and considering stable semantics, as $p m$ (Nixon) holds in each minimal model, it is concluded that this fact holds.

In our approach, according to our generic scenario, and assuming that the sources are not aware of the rules, $\langle p m(N$ ixon $), \mathrm{b}\rangle$ belongs to the semantics of the integrated database, because $\langle$ dove(Nixon), b $\rangle$ and $\langle$ hawk(Nixon), b $\rangle$ are also in the semantics of this database.

However, if we now assume that the sources are aware of the rules, then they are defined by $S_{1}^{\prime \prime}=S_{1}^{\prime} \cup\{\langle p m($ Nixon $), \mathrm{t}\rangle\}$ and $S_{2}^{\prime \prime}=S_{2}^{\prime} \cup\{\langle p m($ Nixon $), \mathrm{t}\rangle\}$. Then, contrary to the previous case, $\langle p m(N$ ixon $), t\rangle$ belongs to the semantics of the integrated database $\Delta^{\prime \prime}=\left(E^{\prime \prime}, R\right)$, because this pair is in $E^{\prime \prime}$.

This example shows that, when integrating data in a deductive context, deduction and integration do not commute in general. A generic scenario in this respect would assume that each data source has its own facts and rules, whereas the integration site has its own rules. Then, in this context, data integration can be processed according to the following distinct policies: 
1. Data and rules are collected from each source and deductions are computed in the integration site using the integrated data and all rules obtained from the data sources.

2. Only data are collected from data sources, assuming that each data source has applied its own rules beforehand. Then, further deductions are computed to the integrated data, using the rules available in the integration site.

Although it is likely that these policies give different results, we notice that the second one is computationally easier for the integration site. It is thus relevant to identify cases where commutativity (or a weak but acceptable form of commutativity) holds, a basic issue left to future work.

\subsection{The Trial Example of [13]}

This example is about integrating data coming from two sources meant to represent respectively a prosecutor and a lawyer. Given a person $\lambda$ put on trial, both sources provide information to the judge, the prosecutor trying to convince the judge that $\lambda$ is suspect and the lawyer trying to convince the judge that $\lambda$ is innocent. The judge then integrates this information and decides on charging or not $\lambda$. A simplified version of this process is expressed in [13] as follows.

$$
\begin{aligned}
\text { suspect }(x) & \leftarrow \operatorname{motive}(x) \vee \text { witness }(x) \\
\text { innocent }(x) & \leftarrow \operatorname{alibi}(x, y) \wedge \neg \text { friends }(x, y) \\
\text { friends }(x, y) & \leftarrow \operatorname{friends}(y, x) \vee(\text { friends }(x, z) \wedge \operatorname{friends}(z, y)) \\
\operatorname{charge}(x) & \leftarrow \operatorname{suspect}(x) \oplus \neg \text { innocent }(x)
\end{aligned}
$$

Here, the syntax for the rules is that of Fitting programs ([7]) where the connectors $\vee, \wedge, \oplus$ (maximum with respect to $\preceq_{k}$ ) and $\otimes$ (minimum with respect to $\preceq_{k}$ ) appear in the bodies of the rules. This syntax is compatible with our approach because $(i)$ it is well known that the use of $\vee$ allows to group all rules with the same head into one rule, and (ii) allowing the connectors $\oplus$ and $\otimes$ in our approach is a sound extension, that is left to future work.

To clarify the intuitive meaning of the last rule, we explain how the judge decides on charging a person $\lambda$ :

- If either suspect $(\lambda)$ or $\neg$ innocent $(\lambda)$ is true while the other is neither false nor contradictory, then $\lambda$ is charged.

- If suspect $(\lambda)$ and $\neg$ innocent $(\lambda)$ are contradictory (one is true and the other is false, or one is contradictory), then charge $(\lambda)$ is also contradictory, meaning that the trial needs to be refined, because one of the sources is lying...

- If these two formulas are unknown, then so is also the fact that $\lambda$ is charged. In this case more information is needed to reach a conclusion.

As in [13], but in our formalism, assume that the prosecutor and the lawyer respectively assert that $\langle$ witness(John), f $\rangle$ and $\langle$ friends $(J o h n, T e d), \mathrm{t}\rangle$ hold. According to our integration scenario, in the integrated database $\Delta=(E, R)$, $E$ contains these two pairs, thus implying that charge(John) is unknown in $\Delta$.

In [13], the authors show how additional knowledge, called hypotheses, can be checked against such database. For example, it is shown that assuming that 
innocent(John) is true and that charge(John) is false constitute such a compatible set, and so, that not charging John is consistent.

Although, in our approach, the goal is different, since we look for deriving information from the given program, independently from any hypothesis, the work of [13] can be stated by: given $\Delta=(E, R)$ and hypotheses $H$ (as a set of pairs), does $\Delta$ have a model containing $H$ ? For instance in our case, with $H=\{\langle$ innocent $(\operatorname{John}), \mathrm{t}\rangle,\langle\operatorname{charge}(\operatorname{John}), \mathrm{f}\rangle\}$, it can be seen that the answer is yes, thus leading to the same conclusion as in [13].

On the other hand, as our approach assumes OWA, no false facts can be derived, unless explicitly stated in the program. We point out in this respect that assuming CWA is not appropriate in the present context. Indeed, assume that alibi(John,Ted) is stored as true and that nothing is known about John's friends. In this case, CWA allows to state that $\neg$ friends (John,Ted) holds, thus that innocent (John) holds as well. However, in order to convince the judge that innocent (John) holds, the lawyer is meant to provide evidence of the fact that friends (John,Ted) is false, which is not modeled in [13].

\section{Conclusion}

In this paper we have introduced a novel approach to deductive databases dealing with contradictory information. This work is motivated by the facts that $(i)$ many contradictions occur in the real world, and (ii) data integration is a field where such contradictions are common. We thus consider a deductive database approach based on the four-valued logics introduced in [3]. Our database semantics has been shown to be 'compatible' with two popular implications $([2,16])$, and some basic properties regarding minimality have been investigated.

As this work is preliminary, many issues are still to be investigated: $(i)$ consider extended rules as defined in [7]; (ii) investigate the use of the two-valued operators introduced in [16] to allow rules expressing sentences such as if $\varphi$ is unknown then $\varphi^{\prime}$ is false; (iii) when rules are Datalog ${ }^{\text {neg }}$ rules, compare our semantics with those of Datalog ${ }^{n e g}([5])$; (iv) integrate standard constraints such as functional dependencies in our approach; $(v)$ design and study a relational algebra dealing with tuples in the four-valued logics framework; and (vi) define a measurement of inconsistency, as in previous work such as [10] or [15].

\section{A Proof of Lemma 1}

Lemma 1. For every $\Delta=(E, R)$ and all v-sets $S, S_{1}$ and $S_{2}$ :

1. $\Sigma_{\Delta}(S)$ is a v-set such that $E \preceq_{k} \Sigma_{\Delta}(S)$.

2. If $S_{1} \preceq_{k} S_{2}$, then $\Sigma_{\Delta}\left(S_{1}\right) \preceq_{k} \Sigma_{\Delta}\left(S_{2}\right)$.

Proof. 1. $\Sigma_{\Delta}(S)$ is a v-set because $\mathrm{v}_{\oplus}(\varphi)$ is unique, for a fixed $\varphi$. Moreover, every $\langle\varphi, \mathrm{v}\rangle$ in $E$ also belongs to $\Gamma_{\Delta}^{E}(S)$, and as no rule can generate another pair involving $\varphi,\langle\varphi, \mathrm{v}\rangle$ is the only pair of $\Gamma_{\Delta}^{E}(S)$ involving $\varphi$. Hence $\langle\varphi, \mathrm{v}\rangle$ is in $\Sigma_{\Delta}(S)$, and so $E \subseteq \Sigma_{\Delta}(S)$ implying that $E \preceq_{k} \Sigma_{\Delta}(S)$. 
2. Let $v_{1}$ and $v_{2}$ denote respectively the valuations defined by $\Sigma_{\Delta}\left(S_{1}\right)$ and $\Sigma_{\Delta}\left(S_{2}\right)$, and let $\langle\varphi, \mathrm{v}\rangle$ be in $\Sigma_{\Delta}\left(S_{1}\right)$. If $\varphi$ occurs in $E$, then the previous point shows that $\langle\varphi, \mathrm{v}\rangle$ also belongs to $\Sigma_{\Delta}\left(S_{2}\right)$, and so $v_{1}(\varphi)=v_{2}(\varphi)$. Assuming now that $\varphi$ does not occur in $E$ implies that rules whose head is $\varphi$ or $\neg \varphi$ have generated pairs involving $\varphi$. We consider the following distinct cases:

- Case 1: $v_{1}(\varphi)=\mathrm{t}$ or $v_{1}(\varphi)=\mathrm{f}$. Since in $S_{1}, \mathrm{v}_{\oplus}(\varphi)=\mathrm{t}$ (respectively $\mathrm{v}_{\oplus}(\varphi)=\mathrm{f}$ ), $\Gamma_{\Delta}^{E}\left(S_{1}\right)$ contains $\langle\varphi, \mathrm{t}\rangle$ (respectively $\langle\varphi, \mathrm{f}\rangle$ ) and not $\langle\varphi, \mathrm{b}\rangle$. Then, by Definition $4(1)$, inst $(E, R)$ contains a rule $\rho$ such that head $(\rho)=\varphi$ (respectively $\operatorname{head}(\rho)=\neg \varphi)$ and $v_{S_{1}}(\operatorname{bod} y(\rho))=\mathrm{t}$. Then, for every $l$ in $\operatorname{body}(\rho)$, if $l=\phi$, $v_{S_{1}}(\phi)=\mathrm{t}$ and if $l=\neg \phi, v_{S 1}(\phi)=\mathrm{f}$. Since $S_{1} \preceq_{k} S_{2}$, for every $\phi$ occurring in $\operatorname{body}(\rho)$, either $v_{S_{2}}(\phi)=v_{S_{1}}(\phi)$ or $v_{S_{2}}(\phi)=\mathrm{b}$. Hence, whatever the chosen implication $(\rightarrow$ or $\hookrightarrow), v_{2}(\varphi)=v_{1}(\varphi)$ or $v_{2}(\varphi)=\mathrm{b}$ and so, $v_{1}(\varphi) \preceq_{k} v_{2}(\varphi)$.

- Case 2: $v_{1}(\varphi)=\mathrm{b}$. Either $\langle\varphi, \mathrm{b}\rangle$ is in $\Gamma_{\Delta}^{E}\left(S_{1}\right)$ or not.

(i) If $\langle\varphi, \mathrm{b}\rangle$ is in $\Gamma_{\Delta}^{E}\left(S_{1}\right)$, then $\operatorname{inst}(E, R)$ contains $\rho$ such that head $(\rho)=\varphi$ or $\operatorname{head}(\rho)=\neg \varphi$, and $v_{S_{1}}(\operatorname{bod} y(\rho))=\mathrm{b}$. Hence, for every $l$ in $\operatorname{body}(\rho)$, if $l=\phi$, $v_{S_{1}}(\phi)$ is $\mathrm{t}$ or $\mathrm{b}$ and if $l=\neg \phi, v_{S 1}(\phi)$ is $\mathrm{f}$ or $\mathrm{b}$, and at least one of these values is b. Since $S_{1} \preceq_{k} S_{2}, v_{S_{2}}(\phi)$ is either t or b when $v_{S_{1}}(\phi)$ is t or b, and $v_{S_{2}}(\phi)$ is either $\mathrm{f}$ or $\mathrm{b}$ when $v_{S_{1}}(\phi)$ is $\mathrm{f}$ or $\mathrm{b}$, and at least one of these values is $\mathrm{b}$. Therefore $\langle\varphi, \mathrm{b}\rangle$ belongs to $\Gamma_{\Delta}^{E}\left(S_{2}\right)$ and so, in $S_{2}, \mathrm{v}_{\oplus}(\varphi)=\mathrm{b}$. Thus, $v_{1}(\varphi) \preceq_{k} v_{2}(\varphi)$.

(ii) If $\langle\varphi, \mathrm{b}\rangle$ is not in $\Gamma_{\Delta}^{E}\left(S_{1}\right)$, then $\langle\varphi, \mathrm{t}\rangle$ and $\langle\varphi, \mathrm{f}\rangle$ both belong to $\Gamma_{\Delta}^{E}\left(S_{1}\right)$, which implies that $\langle\varphi, \mathrm{b}\rangle$ is in $\Sigma_{\Delta}\left(S_{1}\right)$. Hence $\operatorname{inst}(E, R)$ contains one rule whose head is $\varphi$ and one rule whose head is $\neg \varphi$, and these rules apply for computing $\Gamma_{\Delta}^{E}\left(S_{1}\right)$ and for $(i)$ above. Thus, in $S_{2}$ we have $\mathrm{v}_{\oplus}(\varphi)=\mathrm{b}$, showing that $v_{1}(\varphi) \preceq_{k} v_{2}(\varphi)$. We have shown that for every $\varphi$ occurring $\Sigma_{\Delta}\left(S_{1}\right), v_{1}(\varphi) \preceq_{k} v_{2}(\varphi)$. Now, for every $\varphi$ not occurring in $\Sigma_{\Delta}\left(S_{1}\right), v_{1}(\varphi)=\mathrm{n}$ which is the lowest truth value. Thus, $v_{1}(\varphi) \preceq_{k} v_{2}(\varphi)$ holds, showing that $\Sigma_{\Delta}\left(S_{1}\right) \preceq_{k} \Sigma_{\Delta}\left(S_{2}\right)$ holds as well. Therefore, the proof is complete.

\section{B Proof of Proposition 1}

Proposition 1. Given a database $\Delta=(E, R), \Sigma_{\Delta}^{*}$ is a minimal (with respect to set inclusion) model of $\Delta$.

Proof. If $\Sigma_{\Delta}^{*}$ is not a model of $\Delta$, then one rule $\rho$ of $\operatorname{inst}(E,(R)$ is not valid in $\Sigma_{\Delta}^{*}$. In this case, head $(\rho)$ is not valid, while the conjunct defined by $\operatorname{bod} y(\rho)$ is valid. Denoting head $(\rho)$ by $\varphi$ (respectively $\neg \varphi$ ), either $v_{\Delta}(\varphi)=\mathrm{n}$ or $v_{\Delta}(\varphi)=\mathrm{f}$ (respectively $v_{\Delta}(\varphi)=\mathrm{t}$ ). If $v_{\Delta}(\varphi)=\mathrm{n}$, since $\operatorname{body}(\rho)$ is valid in $\Sigma_{\Delta}^{*}$, we have $\Sigma_{\Delta}\left(\Sigma_{\Delta}^{*}\right) \neq \Sigma_{\Delta}^{*}$, which is not possible. Therefore, either head $(\rho)=\varphi$ and $v_{\Delta}(\varphi)=\mathrm{f}$, or head $(\rho)=\neg \varphi$ and $v_{\Delta}(\varphi)=\mathrm{t}$, which is not possible by Definition 4 . Thus, $\Sigma_{\Delta}^{*}$ is a model of $\Delta$.

To show that $\Sigma_{\Delta}^{*}$ is a minimal model, let $\sigma$ be a nonempty subset of $\Sigma_{\Delta}^{*}$, and assume that $S=\Sigma_{\Delta}^{*} \backslash \sigma$ is a model of $\Delta$. Let $k$ be the least integer such that $\Sigma^{k-1} \cap \sigma=\emptyset$ and $\Sigma^{k} \cap \sigma \neq \emptyset$. We notice that $k$ exists such that $k>0$ because, since $S$ is a model of $\Delta$, it holds that $E \subseteq S$ and so, since $\Sigma^{0}=E$, we have $\Sigma^{0} \cap \sigma=\emptyset$. Now, let $\langle\varphi, \mathrm{v}\rangle$ be in $\Sigma^{k} \cap \sigma$ but not in $\Sigma^{k-1}$. In this case, $v_{S}(\varphi)=\mathrm{n}$ and as above, there exists one rule $\rho$ in $\operatorname{inst}(E, R)$ such that head $(\rho)$ 
is either $\varphi$ or $\neg \varphi$ and in $\Sigma^{k-1}, \operatorname{head}(\rho)$ is not valid, while the conjunct defined by $\operatorname{body}(\rho)$ is valid. Since $\Sigma^{k-1} \subseteq S$, $\operatorname{body}(\rho)$ is valid in $S$ while head $(\rho)$ is not. This is a contradiction and so, the proof is complete.

\section{Proof of Proposition 2}

Proposition 2. Let $\Delta=(E, R)$ be such that for every rule $\rho$ in $R$, head $(\rho)$ is a positive literal. For all minimal models $M_{1}$ and $M_{2}$ of $\Delta$, the following holds: (i) $\mathrm{V}\left(M_{1}\right)=\mathrm{V}\left(M_{2}\right)$ and $(i i) \mathrm{F}\left(M_{1}\right)=\mathrm{F}\left(M_{2}\right)$.

Proof. The proposition is a consequence of Lemma 2 shown next.

Lemma 2. Let $\Delta=(E, R)$ be such that for every rule $\rho$ in $R$, head $(\rho)$ is a positive literal. For every minimal model $M$ of $\Delta$, the following holds:

1. $\mathrm{F}\left(\Sigma_{\Delta}^{*}\right)=\mathrm{F}(M)$.

2. $\mathrm{V}\left(\Sigma_{\Delta}^{*}\right)=\mathrm{V}(M)$.

Proof. 1. As computing $\Sigma_{\Delta}^{*}$ starts from $E$ and generates no other false facts, $\mathrm{F}\left(\Sigma_{\Delta}^{*}\right)=\mathrm{F}(E)$. Since $\mathrm{F}(E) \subseteq \mathrm{F}(M)$, we obtain $\mathrm{F}\left(\Sigma_{\Delta}^{*}\right) \subseteq \mathrm{F}(M)$.

Assuming that $\mathrm{F}(M) \nsubseteq \mathbb{\mathrm { F }}\left(\Sigma_{\Delta}^{*}\right)$, let $\varphi$ be in $\mathrm{F}(M) \backslash \mathrm{F}\left(\Sigma_{\Delta}^{*}\right)$. Denoting by $M^{\prime}$ the set $M \backslash\{\langle\varphi, f\rangle\}$, we show that $M^{\prime}$ is a model of $\Delta$ and thus that we obtain a contradiction since $M$ is assumed to be minimal. To show that $M^{\prime}$ is a model of $\Delta$, we first note that $E \subseteq M^{\prime}$ holds because so does $E \subseteq M$ and $\langle\varphi, f\rangle$ is not $E$. Thus, assuming that $M^{\prime}$ is not a model of $\Delta$ entails that there exists $\rho$ in $\operatorname{inst}(E, R)$ that is not valid in $M^{\prime}$. Hence, independently from the chosen implication $\rightarrow$ or $\hookrightarrow, \operatorname{bod} y(\rho)$ is valid in $M^{\prime}$ whereas head $(\rho)$ is not. However, since $\operatorname{body}(\rho)$ is valid in $M^{\prime}, \varphi$ does not occur in $\operatorname{body}(\rho)$, implying that body $(\rho)$ is also valid in $M$. Hence, head $(\rho)$ must be valid in $M$ and so, $\operatorname{head}(\rho)=\varphi$. This is a contradiction with the fact that $\varphi$ is assumed to be in $\mathrm{F}(M)$.

2. We prove that $\mathrm{V}\left(\Sigma_{\Delta}^{*}\right) \subseteq \mathrm{V}(M)$ by induction on $k$. Indeed, $\mathrm{V}\left(\Sigma^{0}\right) \subseteq \mathrm{V}(M)$ holds because $E=\Sigma^{0}$. Then, for $k>0$, assume that $\mathrm{V}\left(\Sigma^{k-1}\right) \subseteq \mathrm{V}(M)$ and let $\varphi$ be in $\mathrm{V}\left(\Sigma^{k}\right) \backslash \mathrm{V}(M)$. Since $\mathrm{V}(E) \subseteq \mathrm{V}(M), \varphi$ is not in $\mathrm{V}(E), \varphi$ occurs in $\Sigma^{k}$ due to a rule $\rho$. Thus, there exists $\rho$ in $\operatorname{inst}(E, R)$ such that $\operatorname{body}(\rho)$ is valid in $\Sigma^{k-1}$ and $h e a d(\rho)=\varphi$. Since $\mathrm{V}\left(\Sigma^{k-1}\right) \subseteq \mathrm{V}(M)$, body $(\rho)$ is valid in $M$ and as $\rho$ must be valid in $M$, so is $\varphi$. We thus obtain a contradiction with the fact that $\varphi$ is assumed not to be in $\mathrm{V}(M)$. Hence $\mathrm{V}\left(\Sigma^{k}\right) \subseteq \mathrm{V}(M)$ and thus, $\mathrm{V}\left(\Sigma_{\Delta}^{*}\right) \subseteq \mathrm{V}(M)$.

Now let $M^{\prime}=\left\{\langle\varphi, \mathrm{v}\rangle \in M \mid \varphi \in \mathrm{V}\left(\Sigma_{\Delta}^{*}\right)\right\} \cup\left\{\langle\varphi, \mathrm{f}\rangle \in M \mid \varphi \in \mathrm{F}\left(\Sigma_{\Delta}^{*}\right)\right\}$. We notice that $M^{\prime} \subseteq M$, and since $\mathrm{V}\left(\Sigma_{\Delta}^{*}\right) \subseteq \mathrm{V}(M)$ and $\mathrm{F}\left(\Sigma_{\Delta}^{*}\right)=\mathrm{F}(M)$, we have $\mathrm{V}\left(\Sigma_{\Delta}^{*}\right)=\mathrm{V}\left(M^{\prime}\right)$ and $\mathrm{F}\left(\Sigma_{\Delta}^{*}\right)=\mathrm{F}\left(M^{\prime}\right)$. We show that $M^{\prime}$ is a model of $\Delta$ and thus that $M^{\prime}=M$ since $M$ is assumed to be minimal. To show that $M^{\prime}$ is a model of $\Delta$, we first prove that $E \subseteq M^{\prime}$. Indeed, as every $\langle\varphi, \mathrm{v}\rangle$ in $E$ is also in $\Sigma_{\Delta}^{*}$ and in $M$, we have the following:

- If $\mathrm{v}=\mathrm{f}$ then $\varphi$ is in $\mathrm{F}(E)$ thus in $\mathrm{F}\left(\Sigma_{\Delta}^{*}\right)$. In this case, $\langle\varphi, \mathrm{v}\rangle$ is in $M^{\prime}$.

- Otherwise, $\mathrm{v}=\mathrm{t}$ or $\mathrm{v}=\mathrm{b}$, that is $\varphi$ is in $\mathrm{V}(E)$. Thus $\varphi$ is in $\mathrm{V}\left(\Sigma_{\Delta}^{*}\right)$ and as $\langle\varphi, \mathrm{v}\rangle$ is in $M,\langle\varphi, \mathrm{v}\rangle$ is also in $M^{\prime}$.

Every rule $\rho$ in $\operatorname{inst}(E, R)$ is valid in $M^{\prime}$, because if $\operatorname{body}(\rho)$ is valid in $M^{\prime}$ then $\operatorname{bod} y(\rho)$ is also valid in $\Sigma_{\Delta}^{*}$ and so, head $(\rho)$ is in $\mathrm{V}\left(\Sigma_{\Delta}^{*}\right)$. Thus head $(\rho)$ is in $\mathrm{V}\left(M^{\prime}\right)$. Hence, $M^{\prime}=M$, showing that $\mathrm{V}\left(\Sigma_{\Delta}^{*}\right)=\mathrm{V}(M)$. 


\section{References}

1. Foto N. Afrati and Phokion G. Kolaitis. Repair checking in inconsistent databases: algorithms and complexity. In Ronald Fagin, editor, Database Theory - ICDT 2009, 12th International Conference, Proceedings, pages 31-41, 2009.

2. Ofer Arieli and Arnon Avron. The Value of the Four Values. Artif. Intell., 102(1):97-141, 1998.

3. Nuel D. Belnap. A useful four-valued logic. In Modern Uses of Multiple-Valued Logic. D. Reidel, 1977.

4. Mike Bergman. The Open World Assumption: Elephant in the room. In AI3:::Adaptative Information, pages 1-11. Available at: www.mkbergman.com/852/ the-open-world-assumption-elephant-in-the-room/, 2009.

5. Nicole Bidoit. Negation in rule-based database languages: A survey. Theor. Comput. Sci., 78(1):3-83, 1991.

6. Stefano Ceri, Georg Gottlob, and Letizia Tanca. Logic Programming and Databases. Springer, 1990.

7. Melvin C. Fitting. Bilattices and the Semantics of Logic Programming. J. Log. Program., 11:91-116, 1991.

8. Michael Gelfond, and Vladimir Lifschitz. The stable model semantics for logic programming. In Logic Programming, Proceedings of the International Conference and Symposium, pages 1070-1080, 1988.

9. Gianluigi Greco, Sergio Greco, and Ester Zumpano. A logical framework for querying and repairing inconsistent databases. IEEE Trans. Knowl. Data Eng., 15(6):1389-1408, 2003.

10. John Grant and Antony Hunter. Analysing inconsistent first-order knowledgebases. Artificial Intelligence, 172(8-9):1064-1093, 2008.

11. Sergio Greco, Cristian Molinaro, and Irina Trubitsyna. Computing Approximate Query Answers over Inconsistent Knowledge Bases. In Proceedings of the International Joint Conference on Artificial Intelligence, IJCAI, pages 1838-1846, 2018.

12. Allen P. Hazen and Francis Jeffry Pelletier. K3, L3, LP, RM3, A3, FDE: How to Make Many-Valued Logics Work for You. CoRR, abs/1711.05816, 2017.

13. Yann Loyer, Nicolas Spyratos, and Daniel Stamate. Hypothesis-based semantics of logic programs in multivalued logics. ACM Trans. Comput. Log., 5(3):508-527, 2004.

14. Raymond Reiter. On closed world data bases. In Logic and Data Bases, pages 55-76, 1977.

15. Matthias Thimm. On the Expressivity of Inconsistency Measures (Extended Abstract). In Proceedings of the International Joint Conference on Artificial Intelligence, IJCAI, pages 5070-5074, 2017.

16. Alexis Tsoukiàs. A First-order, Four-valued, Weakly Paraconsistent Logic and its Relation with Rough Sets Semantics. Foundations of Computing and Decision Sciences, 12:85-108, 2002. 